\title{
The influence of substrate temperature on the properties of aluminum-doped zinc oxide thin films deposited by DC magnetron sputtering
}

\author{
Yeon-Keon Moon · Se-Hyun Kim · Jong-Wan Park
}

Received: 2 December 2005/ Accepted: 7 August 2006/ Published online: 7 September 2006

(C) Springer Science+Business Media, LLC 2006

\begin{abstract}
Transparent, conducting, aluminum-doped zinc oxide (AZO) thin films were deposited on Corning 1737 glass by a DC magnetron sputter. The structural, electrical, and optical properties of the films, deposited using various substrate temperatures, were investigated. The AZO thin films were fabricated with an AZO ceramic target $\left(\mathrm{Al}_{2} \mathrm{O}_{3}: 2 \mathrm{wt} \%\right)$. The obtained films were polycrystalline with a hexagonal wurtzite structure and preferentially oriented in the (002) crystallographic direction. The lowest resistivity was $6.0 \times 10^{-4} \Omega \mathrm{cm}$, with a carrier concentration of $2.7 \times 10^{20} \mathrm{~cm}^{-3}$ and a Hall mobility of $20.4 \mathrm{~cm}^{2} / \mathrm{Vs}$. The average transmittance in the visible range was above $90 \%$.
\end{abstract}

\section{Introduction}

Transparent, conductive oxide (TCO) films, primarily indium tin oxide (ITO) films, have been widely applied to the manufacturing of transparent electrodes for flat panel displays, solar cells, organic, light-emitting diodes, integrated optics, piezoelectronic, gas sensor, heat mirror, and surface acoustic wave (SAW) devices due to their high luminous transmittance, good electrical conductivity, good adhesion to substrates, and chemical inertness. However, the high cost of ITO films has motivated efforts to develop alternatives. In

Y.-K. Moon $(\bowtie) \cdot$ S.-H. Kim · J.-W. Park

Division of Materials Science and Engineering, Hanyang

University, 17 Haengdang-Dong, Seongdong-Ku, Seoul 133-

791, Korea

e-mail: opendoor79@hanyang.ac.kr particular, aluminum-doped zinc oxide (AZO) thin films, the n-type dopant, have attracted attention as TCO films because of their low resistivity and high transmittance in the visible range. $\mathrm{ZnO}$-based TCOs are relatively inexpensive, and $\mathrm{ZnO}$-based materials also have desirable properties, such as non-toxicity, long-term environmental stability, and excellent infrared ray (IR) shielding [1].

AZO thin films can be produced by various deposition techniques, including pulsed laser deposition [2], chemical vapor deposition [3], sol-gel process [4], spray pyrolysis [5], evaporation [6], and magnetron sputtering [7]. Among the methods, magnetron sputtering has several advantages, such as low processing temperature, good adhesion of films on substrates, very good thickness uniformity, high deposition rates, high density of the films, and the relative ease of scaling to large areas. It is also a simple process, and the process parameters are easy to control.

In this paper, we investigated the influence of substrate temperature on the structural, electrical, and optical properties of AZO thin films, deposited on Corning 1737 glass substrates. AZO thin films were fabricated by DC magnetron sputtering with an AZO ceramic target $\left(\mathrm{Al}_{2} \mathrm{O}_{3}: 2 \mathrm{wt} \%\right)$.

\section{Experimental}

AZO thin films, deposited on Corning glass 1737 (thickness: $0.7 \mathrm{~mm}$ ) were prepared using a DC magnetron sputtering system. The targets used in this study were sintered stoichiometric $\mathrm{ZnO}: \mathrm{Al}$ (99.999\% purity, $4 \mathrm{~mm}$ in diameter, $0.5 \mathrm{~mm}$ in thickness). The content of $\mathrm{Al}_{2} \mathrm{O}_{3}$ added to the $\mathrm{ZnO}$ powder target ranged from 
$2 \mathrm{wt} \%$. The glass substrates were ultrasonically cleaned in acetone and methanol and rinsed in an ultrasonic bath in deionized water for $10 \mathrm{~min}$, with subsequent drying by nitrogen gas flow before deposition. The sputtering was performed in an Ar atmosphere with a target to substrate distance of $40 \mathrm{~mm}$. An oil diffusion pump with a rotary pump was used to achieve $2.0 \times 10^{-6}$ mTorr pressure before introducing the Ar gas. The substrate temperature was measured by a thermocouple gauge and a hot cathode gauge. The variation in substrate temperature during the deposition process was maintained within $\pm 5^{\circ} \mathrm{C}$. The substrate temperatures used in AZO film deposition ranged from room temperature (R.T.) to $500^{\circ} \mathrm{C}$.

The structural, electrical, and optical properties of the AZO thin films were characterized by various techniques. The film thickness was measured, using a surface profiler (Alpha-Step 500, TENCOR, USA) and a field emission scanning electron microscope (FE-SEM, S-4300 Hitachi). A high-resolution X-ray diffractometer (HR-XRD, Bruker D8 DISCOVER, Germany) was used to investigate the crystallinity and crystal orientation of the films. The surface morphology and the surface roughness of the AZO films were examined by atomic force microscopy (AFM, AP0190, Auto-Probe CP Multitask Microscopy). The electrical resistivity, carrier concentration, and Hall mobility were measured at R.T. by the Van der Pauw method. The optical transmittance of the AZO films was measured in the visible range, using an UV-vis NIR spectrophotometer (Japan Shimadzu UV-3101PC). The elemental compositions were investigated by Rutherford back scattering (RBS, NEC 53DH-2) and secondary ion mass spectroscopy (SIMS, PH17200 TOF-SIMS).

\section{Results and discussion}

\subsection{Crystallinity properties}

The thin AZO films were deposited at $40 \mathrm{~W}$ and 5 mTorr with various substrate temperatures, ranging from R.T. to $500^{\circ} \mathrm{C}$. The effects of substrate temperature on the microstructure of AZO thin films were investigated through XRD analysis. Figure 1 shows the X-ray diffraction patterns of the AZO films. As the substrate temperature increased, the peak intensity increased. The peak intensity is the largest at the substrate temperature of $300^{\circ} \mathrm{C}$; then, as the substrate temperature increased above $300^{\circ} \mathrm{C}$, the peak began to decrease. These trends were caused by a different level of incident energy at the substrate at various

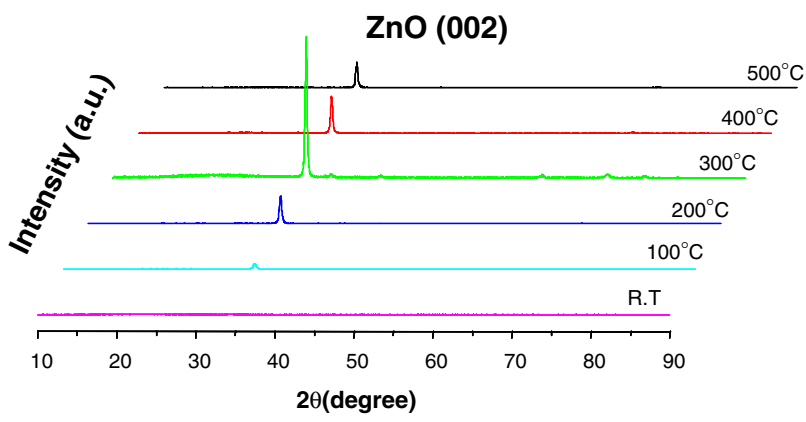

Fig. 1 X-ray diffraction patterns for AZO films deposited with different substrate temperatures

temperatures. Also, the vapor pressure of zinc is relatively higher than that of aluminum. This is why the aluminum doping level at a substrate temperature of $300^{\circ} \mathrm{C}$ was higher than at the other substrate temperatures [8]. Figure 2 shows the full width at half maximum (FWHM) and grain size of the AZO films, determined through the use of Scherrer's formula. The grain size was inversely proportional to the FWHM. The largest grain size was $\sim 27 \mathrm{~nm}$ at $300^{\circ} \mathrm{C}$. AFM images are shown in 3 . The RMS roughness of the AZO thin film, deposited at $300^{\circ} \mathrm{C}$, was lower than the other thin films.

\subsection{Electrical properties}

Figure 4 shows the electrical properties of the AZO thin films deposited with various substrate temperatures. The resistivity of the films decreased as the substrate temperature increased from R.T. to $300^{\circ} \mathrm{C}$. The minimum value for the resistivity of $6.0 \times 10^{-4} \Omega \mathrm{cm}$ was obtained at $300^{\circ} \mathrm{C}$. A further increase in substrate temperature resulted in a slight increase of resistivity.

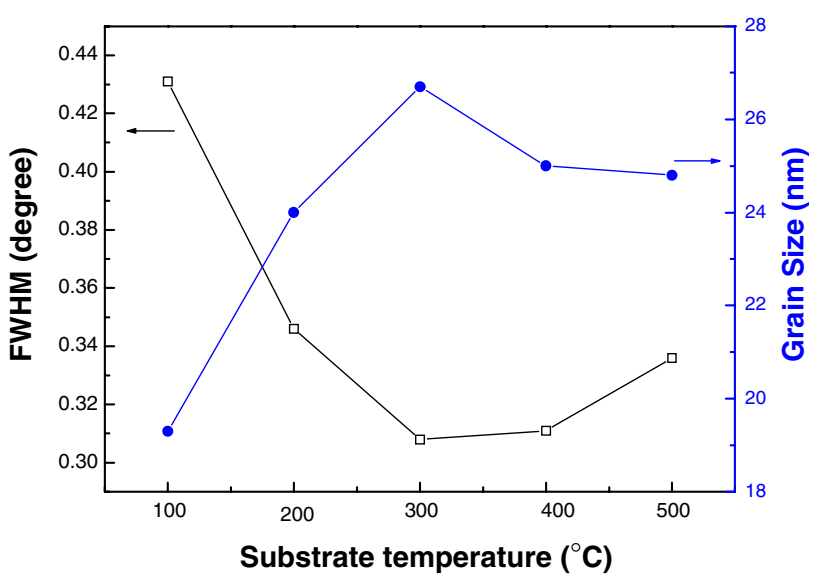

Fig. 2 Full width at half maximum (FWHM) of XRD (002) peaks and the grain sizes for AZO films deposited with various substrate temperatures 


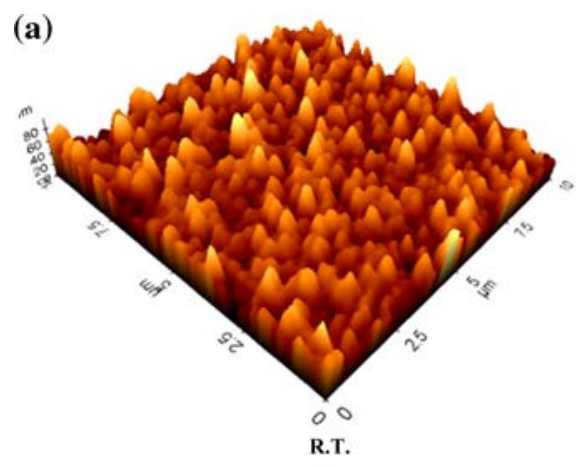

(b)

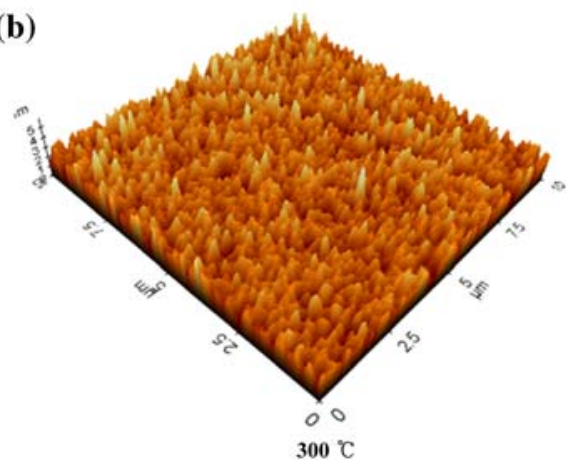

(c)

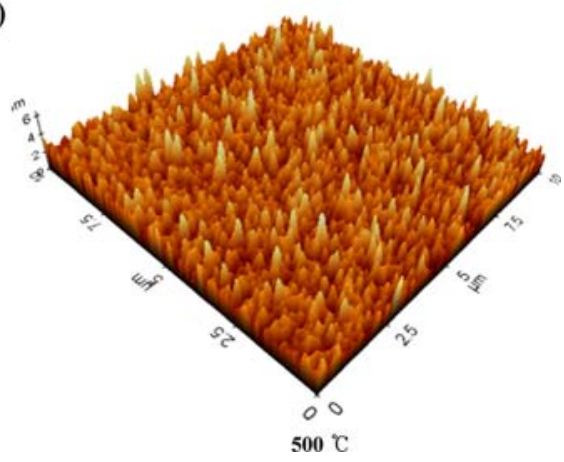

Fig. 3 AFM images of the surface of AZO thin films with various substrate temperatures; (a) R.T., (b) $300^{\circ} \mathrm{C}$, and (c) $500^{\circ} \mathrm{C}$
Both the carrier concentration and Hall mobility increased as the substrate temperature increased from R.T to $300^{\circ} \mathrm{C}$, reaching their maximum values of $2.7 \times 10^{20} \mathrm{~cm}^{-3}$ and $20.4 \mathrm{~cm}^{2} / \mathrm{Vs}$, respectively, at $300^{\circ} \mathrm{C}$. As the substrate temperature increased further, both the carrier concentration and Hall mobility decreased.

\subsection{Optical properties}

The transmittance spectra, in the range of $300-800 \mathrm{~nm}$, for the AZO thin films deposited with various substrate temperatures are shown in 5. The film deposited at R.T has a relatively low transmittance, but it still exhibited a visible light transmittance of about $80 \%$. Also, all films, excluding the thin film deposited at R.T., exhibited average transmittances above $90 \%$ in the visible region. Figure 6 shows plots of $(\alpha \mathrm{h} v)^{2}$ vs. hv for the AZO films deposited with various substrate temperatures. The absorption edge for direct interband transition is given by Serpone et al. [9],

$\alpha h v=C\left(h v-E_{g}\right)^{1 / 2}$

where $\alpha$ is an absorption coefficient, $h v$ denotes photon energy, $C$ is a constant for a direct transition, and $E_{\mathrm{g}}$ is the optical energy band gap.

The optical energy gap $E_{g}$ can be obtained from the intercept of $(\alpha \mathrm{h} v)^{2}$ vs. $h v$ for direct allowed transition [10]. The optical band gap widths of the AZO films were estimated to be $3.42,3.44,3.59,3.68,3.62$, and $3.60 \mathrm{eV}$ at substrate temperatures of R.T, 100, 200, 300, 400 , and $500^{\circ} \mathrm{C}$, respectively. The absorption edge of the transmittance shifted to the shorter wavelength (blue-shift) region up to the substrate temperature of $300^{\circ} \mathrm{C}$. This result is believed to be due to the
Fig. 4 Electrical resistivity $(\rho)$, carrier concentration $(n)$, and Hall mobility $(\mu)$ of AZO films deposited with various substrate temperatures

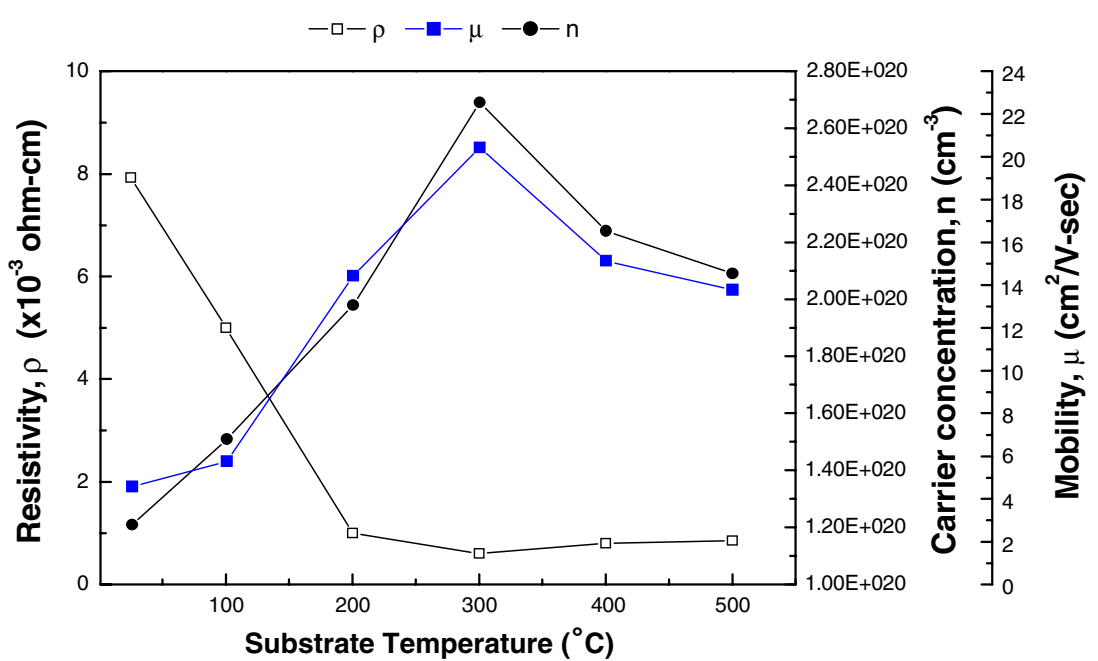




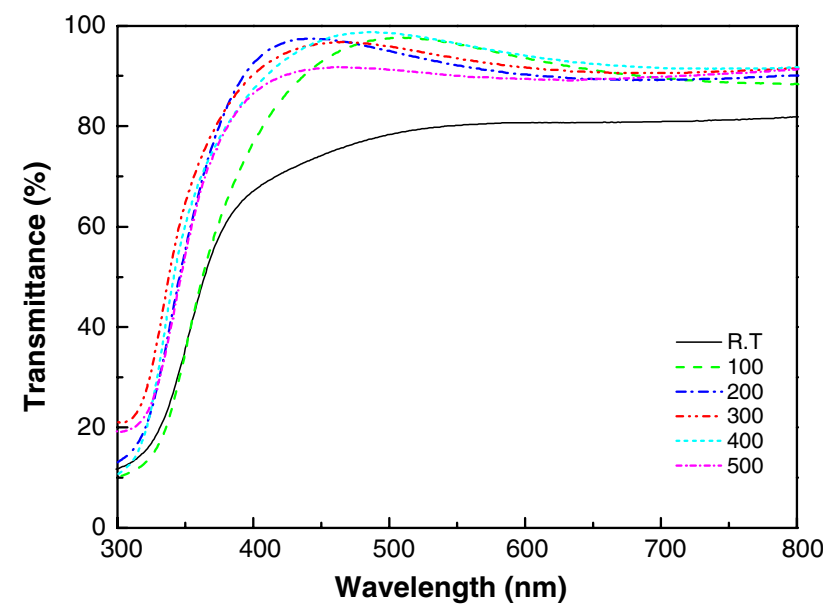

Fig. 5 Optical transmittance spectra of AZO films deposited with various substrate temperatures

Burstein-Moss effect hypothesis. which predicts that the Fermi level inside the conduction band moves upward with increasing donor concentration due to the filling of the conduction band by the increase of electron carriers. It is known that the optical band gap is proportional to the carrier concentration. The optical band gap decreased with increasing substrate temperature above $300^{\circ} \mathrm{C}$. These facts correspond with the aforementioned hypothesis.

\subsection{Structure and surface morphology}

1 shows the RMS roughness values of the AZO thin films deposited with various substrate temperatures. The RMS roughness values were 11.0, 0.59, and $0.73 \mathrm{~nm}$ for the thin films deposited with the substrate temperatures of R.T., $300^{\circ} \mathrm{C}$, and $500^{\circ} \mathrm{C}$, respectively. The increase in surface roughness of the films promotes oxygen absorption on the surface of the crystallites to

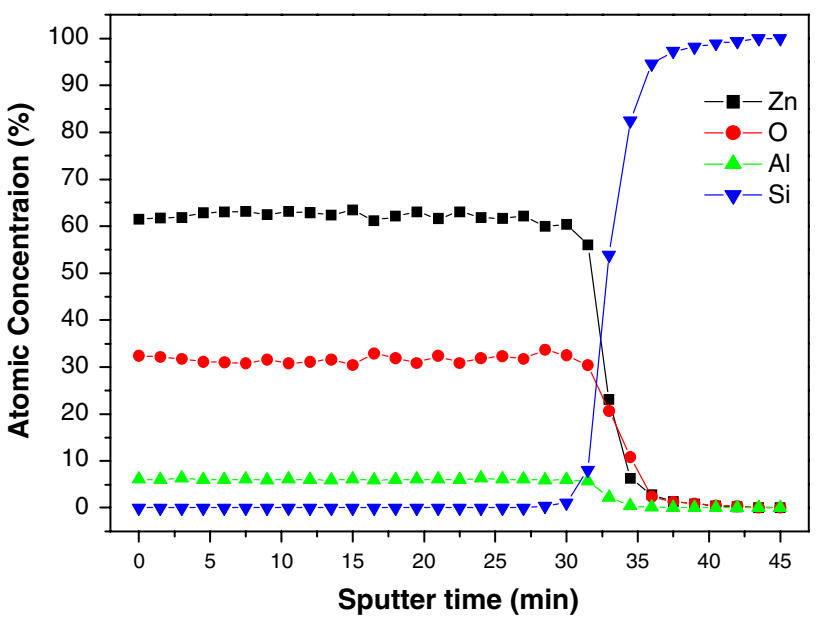

Fig. 7 SIMS depth profiles of AZO films deposited at $300^{\circ} \mathrm{C}$ (with optimization conditions)

form dangling bonds, which then act as electron traps [11]. These electron traps are responsible for the decrease in carrier concentration and Hall mobility. In addition, the rough surface morphology scatters the incident light, thus reducing optical efficiencies. However, the change of morphology with increasing substrate temperature seems to be rather small except for the film deposited at R.T. Therefore, electronic and optical properties of AZO films would not be affected by the surface roughness in the present case.

The compositions of the AZO thin films, determined using SIMS referenced by RBS, are shown in 6 . The analysis shows that AZO films exhibited a homogenous composition, but the ratio of $\mathrm{Zn} / \mathrm{O}$ is, in general, greater than 1 . This suggests non-stoichiometric structural properties of $\mathrm{ZnO}$ [12]. The depth profile of the AZO film resulted in an atomic ratio of $\mathrm{Zn} / \mathrm{O} / \mathrm{Al}$ of $62.27 / 31.46 / 5.93 \%$. The ratio of $\mathrm{Al} / \mathrm{Zn}$, by weight, was $1.1 \%$, which is greater than that in the target
Fig. 6 Plot of $(\alpha \cdot h v)^{2}-h v$ properties of $\mathrm{ZnO}: \mathrm{Al}$ films deposited with various substrate temperatures

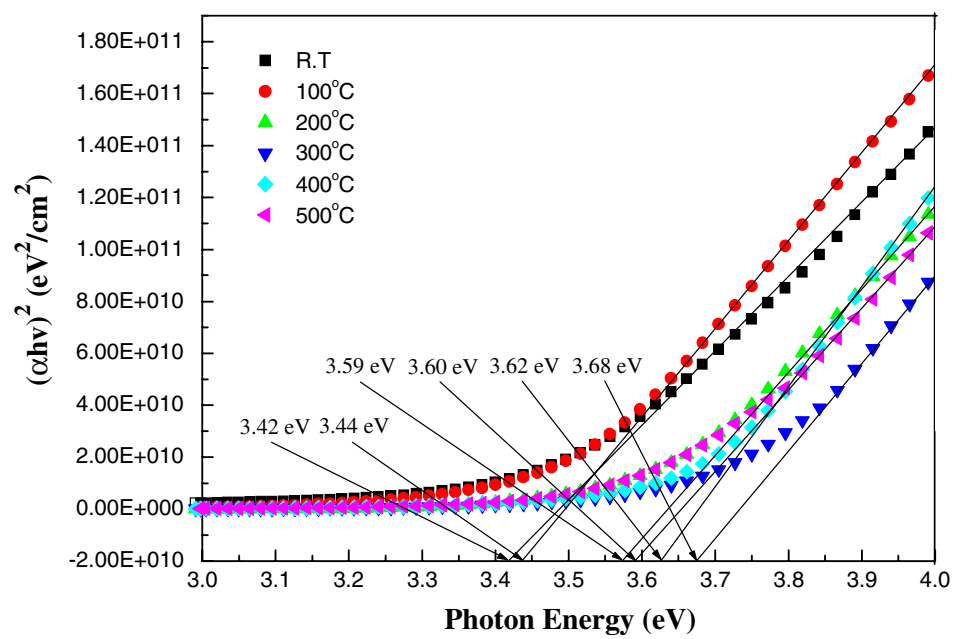

Springer 
Table 1 The surface roughness, measured by AFM, of AZO films prepared with different substrate temperatures

\begin{tabular}{lc}
\hline Substrate temperature $\left({ }^{\circ} \mathrm{C}\right)$ & RMS roughness $(\mathrm{nm})$ \\
\hline R.T. & 11.0 \\
300 & 0.59 \\
500 & 0.73 \\
\hline
\end{tabular}

( $2 \mathrm{wt} \%$ ). This may be due to the sputter yield property of $\mathrm{Zn}$ and $\mathrm{Al}$ atoms [13].

\section{Conclusion}

AZO films have been deposited by DC magnetron sputtering, with a $\mathrm{ZnO}$ target, mixed with $2 \mathrm{wt} \% \mathrm{Al}_{2} \mathrm{O}_{3}$, on Corning 1737 glass. The objective of this study was to investigate the structural, electrical, and optical properties of thin films, deposited with various substrate temperatures. The obtained films were polycrystalline with a hexagonal wurtzite structure and were preferentially oriented in the (002) crystallographic direction. The lowest resistivity was $6.0 \times 10^{-4} \Omega \mathrm{cm}$, with a carrier concentration of $2.7 \times 10^{20} \mathrm{~cm}^{-3}$ and a Hall mobility of $20.4 \mathrm{~cm}^{2} / \mathrm{Vs}$. The average transmittance in the visible light range was above $90 \%$. In this study, we demonstrated the possibility of producing TCO films based on $\mathrm{ZnO}$ with good electrical and optical properties by optimizing the substrate temperature.
Acknowledgements This work was supported by Samsung Electronics Co. through the Brain Korea 21 project under the ministry of Education and Human Resources Development.

\section{References}

1. J.S. Kim, M. Granstrom, R.H. Friend, N. Johansson, W.R. Salaneck, R. Daik, W.J. Feast, F. Cacialli, J. Appl. Phys. 84 6859 (1998)

2. J.H. Kim, K-C. Lee, C. Lee, Proc. SPIE Int. Soc. Opt. Eng. 49, 5063 (2003)

3. T.M. Barnes, S. Hand, J. Leaf, C.A. Wolden, J. Vac. Sci. Technol. A 22, 2118 (2004)

4. M.J. Alam and D.C. Cameron, J. Vac. Sci. Technol. A 19, 1642 (2001)

5. J.M. Bian, X.M. Li, X.D. Gao, W.D. Yu, L.D. Chen Appl. Phys. Lett. 84, 541 (2004)

6. T. Miyata, Y. Minamino, S. Ida, T. Minami, J. Vac. Sci. Technol. A 22, 1711 (2004)

7. B. Huang, S. Wu, J. Li, S. Sun, Z-Q. Tian, Proc. SPIE Int. Soc. Opt. Eng. 152, 5774 (2004)

8. C.-H. Park, H.-J. Lee, H.-B. Kim, G.-H. Lee, J. Kor. Inst. Surf. Eng. 38, 188 (2005)

9. N. Serpone, D. Lawless, R. Khairutdinow J. Phys. Chem. 99, 16646 (1995)

10. I.K. El Zawawi, R.A. Abd Alla, Thin Solid Films 339, 314 (1999)

11. Y. Igasaki, H. Satio, Thin Solid Films 199, 233 (1991)

12. K.L. Chopra, S. Major and D.K. Pandya, Thin Solid Films 102, 1 (1983)

13. M. Ohring, Materials Science of Thin Films, 2nd edn. (Academic), p. 176 (2002) 